\title{
Assessment of adhesive setting time in reinforced concrete beams strengthened with carbon fibre reinforced polymer laminates
}

\author{
Moatasem M. Fayyadh, H. Abdul Razak
}

The dynamic parameters of structural elements have been an area of interest over the last few years, especially with the increased focus on health monitoring and damage detection, which are dependent on the relation between the dynamic and physical properties of structural elements. A great deal of research has focused on the use of dynamic parameters to assess and monitor the structural element performance and stiffness. Some have introduced new damage detection algorithms, whilst others have discovered existing phenomena related to dynamic parameters, structural performance and stiffness $[1,2]$. A hybrid health monitoring system was proposed to detect damage in PSC girder bridges

[3], where the occurrence of damage was alarmed by measuring the change in frequency responses from accelerometers mounted on the PSC girder. Structural health monitoring using statistical time series methods was applied to damage diagnosis in a lightweight truss structure [4], and both non-parametric and parametric methods were shown to effectively tackle damage detection and identification, with parametric methods achieving excellent performance with zero false alarm, missed damage, and damage misclassification rates. A new damage severity algorithm was proposed by [5], which based on the combination of both natural frequencies and mode shape and it was proven to be better sensitive than exist damage severity algorithms. Lower modes were found to be more sensitive to the change in the support conditions [6]. A new damage detection index based on the combination between the mode shape vectors and their curvature was developed and verified to have higher sensitivity than existing algorithms [7].

Carbon Fibre Reinforced Polymer (CFRP) is fast becoming an extensively used material for externally bonded strengthening and repair of Reinforced Concrete (RC) structures. Recent years have seen many researchers using Fibre Reinforced Polymer (FRP) sheets to strengthen and repair RC structures, accompanied by the examination of the parameters affecting the performance of the RC structure when strengthened and repaired with FRP material. Studies have dealt with the configuration of the FRP sheets on the RC structural 
elements and the failure modes of the FRP sheets [8-10]. In[11]

it was found that delamination initiate at the interface from the inner

free edges of the holes and with time, delamination from two

holes meet each other forming a big delamination area and it is evident

that kevlar/epoxy-graphite/epoxy hybrid laminate is more

flexible compared to only graphite/epoxy laminates. The experimental

results carried in [12] demonstrated that composite wrapping

can enhance the structural performance of concrete columns

under axial loading and the number of layers of FRP materials and

the corner radius are the major parameters, having a significant

influence on the behaviour of specimens. In [13], a new model was proposed to predict the intermediate crack

debonding load and it was proven that the proposed model furnishes good predictions,

where the ratio between the IC debonding load predicted by the proposed

model and that experimental furnishes a mean value of

0.9619 and a standard deviation of 0.1062 . The interfacial shear

stresses found to be influenced by the geometry parameters such

as thickness of the FRP plate and adhesive layer in range of the different

degrees [14], where the interfacial shear stress concentrations

and levels increase obviously with the increase of the thickness of

the FRP plate. The cross-sectional shape found to has a significant

influence on the effectiveness of the CFRP-confinement under concentric

loading [15], where member with the circular cross-section

benefited the most, followed by the member with the square

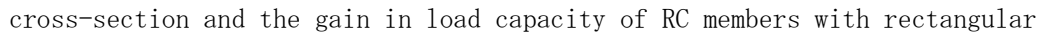

cross-sections due to CFRP-confinement depends on the

aspect ratio of the cross-section.

The first dynamic assessment for the use of FRP plate as an

externally bonded strengthening system using modal testing is

conducted by [16]. Repairing a damaged RC beam with FRP plates

leads to a decrease in the natural frequencies for the first three

bending modes [16]. Dynamic parameters are used to assess the

use of FRP plates to repair damaged RC beams; the bending frequencies

of the repaired phase after exposure to load shows a sma11

increase when compared to the pre-repair damage phase [17]. The

fundamental mode shows lower sensitivity while the third and

fourth modes show higher sensitivity. The modal dampings are

sensitive to both the damaged and the repaired stage [17]. There

is variation in the sensitivity of the bending modes when repairing

the damaged beams with bonded CFRP sheets, depending on the

flexural steel ratio and the pre-repair damage level [18]. The use

of the MAC index shows a relatively smaller change in the damage 
and repair compared to the natural frequencies. This is an inconsistency

which renders the MAC an unreliable index [18]. The fundamental

mode has higher sensitivity to damage and repair [19].

Modal testing conducted on an existing bridge repaired with externally

bonded CFRP sheets shows that both vertical and horizontal

excitation modes are affected, with all the modes experiencing

an increase in the frequencies at the repair phase when compared

to the damage phase $[20]$.

For RC elements repaired with CFRP sheets, the performance and

the stiffness is affected by the properties of the adhesive interface

layer between the CFRP sheets and the RC structure. Some studies

have investigated the effect of the adhesive layer properties on

the structural and dynamic properties of the CFRP repaired RC

structures. The strength of the bonded adhesive reduces due to both

absorption of moisture and an increase in test temperature [21].

Thus, considerable drying may be required to obtain a good bond

[21]. In [22], a method is proposed to estimate the fatigue strength

of the adhesive bonded joint between CFRP sheets and the aluminium

surface. A 3D model to predict the behaviour of the bond between

the CFRP plate and concrete under elevated temperatures

is developed in [23]. The study finds that the adhesive properties

of epoxy are very sensitive to temperature variation. Investigations

have also been conducted with regards to the behaviour of the

adhesive bondage between CFRP sheets and concrete under an impact

loading [24]. Temperature cycles and moisture are associated

with failure in the concrete substrate, while slat fog cycles induce

failure at the concrete-adhesive interface [25]. Immersion in salt

water and salt fog considerably reduces the bond strength [25].

Bond strength increases with an increase in concrete strength and

decreases with an increase in the CFRP plate width [26]. The failure

mode is found to be significantly affected by elevation in temperature

and moisture [27]. In [28], significant effects are observed of

the FRP properties and epoxy curing condition on the interfacial

strength. In [29], the strain distribution is examined along the

bonding line and through the thickness of the adhesive layer and failure mechanisms. In [30], a decrease is observed

in the shear modulus due to creep dependence. It is also shown that beyond a

shear stress corresponding to $40 \%$ of the ultimate bonded strength,

creep is linear.

A11 of the aforementioned works clearly indicate that modal

parameters are affected by the properties and behaviour of RC

beams repaired with CFRP sheets, and mainly by the behaviour 
of the adhesive interface between CFRP sheets and RC structures.

However, no research has been conducted on the use of modal

parameters to investigate the behaviour of the adhesive interface

and to study its properties. Thus, the main objective of this study

is to investigate the setting time behaviour of the adhesive interface

by using the modal parameters and to assess the results based

on the technical data sheets provided by the adhesive material producer.

In addition, this study will show the effect of the setting

time on the adhesive interface using modal parameters to assess

RC structures repaired with CFRP sheets.

\section{Experimental work}

In order to investigate the effect of the adhesive interface setting

time on the modal parameters in RC structures repaired with

CFRP sheets, one RC beam is cast. The RC beam is simply supported

and subjected to flexural loading in order to simulate loading on

actual RC structures such as bridge girders, beams and slabs. The

beam has a clear span length of $2.2 \mathrm{~m}$, a width of $150 \mathrm{~mm}$ and a

depth of $250 \mathrm{~mm}$ in cross section. The dimensions are chosen to

facilitate laboratory testing. The beam details and test setup are

shown in Figs. 1 and 2, and Table 1 details the material properties.

After the beam is cast, it is left for one year to avoid any effect of

the environment or lifetime on the properties of the materials. It

is then tested under point load located at mid-span. Before application

of the load, dynamic properties are obtained using the modal

test and the results are used as data. Load is applied gradually at a

loading rate of $4 \mathrm{kN} / \mathrm{min}$ (i. e. loading and unloading) up to $12 \mathrm{kN}$,

at which point the first crack appears, before decreasing to zero.

Following this all that remains is to recover the deflection and

dynamic properties for the damage case. Roughness equipment is

used on the tension surface to obtain a suitable face and to have

as much friction as possible with the CFRP sheet. Figs. 3 and 4 show

the RC beams before fixing the CFRP sheets and the surface after

roughness. The surface is cleaned using air pressure to avoid any

residue or dust on the surface. This is because the substrate must

be sound, dry, clean and free from laitance, ice, standing water,

grease, oils, old surface treatments or coatings and all loosely adhering particles. The CFRP sheet is fixed using

adhesive material. The CFRP sheet is designed according to ACI 440.2R (2002) [31]

code requirements, as illustrated in the following section, and is

$50 \mathrm{~mm}$ wide, and $1.2 \mathrm{~mm}$ thick while the length is along the clear

span of the beam. The CFRP properties are as shown in Table 2. The

dynamic data is collected half a day after fixing the CFRP sheets 
and is gathered for 18 days.

Full text available at :

http://www.sciencedirect.com/science/article/pii/S0261306911008600 